\title{
Response Analysis of a Plate Structure under Double Source Excitation by FBG Sensors
}

\author{
https://doi.org/10.3991/ijoe.v14i07.8968 \\ Cai Li $(\bowtie)$ \\ Wuhan Donghu University, Wuhan, China \\ caili_whute163.com \\ Huang Xiao \\ Wuhan University of Technology, Wuhan, China \\ Meng Lijun \\ Jianghan University, Wuhan, China
}

\begin{abstract}
The nondestructive assessments play a key role for condition monitoring of in-service structures. At present, many non-destructive testing methods have been widely applied, but actual operation environment has the characteristics of multiple random, which makes the effects of multiple excitation sources. While the traditional detection methods only consider the main sources of motivation and ignore the other sources, which will bring a bigger error. So the multi-source excitation method has practical significance. Here this paper puts forward a double source excitation technique for plate structure based on fiber Bragg grating sensor, which is used to receive the waves in the plate. First, measuring principle of double source excitation and demodulation principle of the FBG sensor are introduced. Then simulation analysis in the double source excitation field is devoted, whose results lay the foundation for the detection in the plate. On this basis, the experimental system is built and the influence of different parameters are analyzed such as frequency, phase. Then this double source excitation-FBG sensing technique is applied to damage detection by envelope analysis, whose result show that this double source excitation-FBG sensing method for the plate structure is sensitive to crack damage.
\end{abstract}

Keywords-Double Source Excitation; Fiber Bragg Grating(FBG) ; Envelope analysis

\section{Introduction}

Currently, the traditional detection methods applied in aircraft structure have certain limitation: On the one hand, damage change of the aircraft structure has the potential and diversity, the existing detection methods are limited lacking of accurate and effective detection method; On the other hand, the existing each kind of detection methods are susceptible to the influence of various interference factors, which are not very sensitive to corresponding structure caused by material mechanics performance 
degradation. In the field of aviation, aircraft structural life depends mainly on material life. Among them, the dosage of aluminum alloy in the aerospace industry is the largest, with high strength, good corrosion resistance, good processability, such outstanding advantages as low cost, it is mainly used for aerospace structure module, wall plate, etc. It may encounter various environment during the running process, and it has the characteristics of multiple random. So the structure response is in fact the effects of multiple excitation source. The traditional detection methods only consider the main sources of motivation and ignore the other sources, which could bring a bigger error. So it has practical significance for the multi-source excitation method.

Multi-source excitation method focus on multi-source vibration response characteristics under different excitation parameters combination. The scholars have gradually tried to study it in the engineering structure detection[1], such as Wieslaw, Junge, and so on. They studied the basis of multi-source excitation problem by changing the position, force. In 2007 the United States Missouri Rolla university, institute of Boeing and air force joint research on the composite damage detection of ultrasonic and eddy current technology. Wang focused on response characteristics of the structure under the multi-point excitation. The structural response can be approximate to monophyletic incentive of superposition of structural response, and the incentives of phase difference have a certain influence on radiation field[2]. Composite technology is gradually becoming a new direction in the field of testing making full use of their methods of advantage to avoid the limitations of single detection technique. Lin studied Hilbert-Huang transform based signal reconstruction technique for the modal analysis of structural systems using vibration measurements. Reliable evaluation of systems' characteristics leads to accurate prediction of systems' behaviors for structural safety purpose[3]. Kashyap presents an analytical model for doubly clamped piezoelectric energy harvesters with segmented electrodes, considering the effect of strain nodes formation and charges cancellation issues, and the analytical solutions are found to closely agree with the Finite Element Method (FEM) simulation results obtained [4]. Adhikari focus on using a stack configuration and harvesting broadband vibration energy, a more practically available ambient source. The mean power acquired from a piezoelectric vibration-based energy harvester subjected to random base excitation is derived using the theory of random vibrations[5]. TianLi Huang studied Hilbert-Huang transform in the spectral representation of earthquake accelerograms, whose results showed that the orthogonal Hilbert spectrum and the orthogonal Hilbert marginal spectrum could produce more faithful representation in characterization of the energy distribution at different frequency regions[6]. H. Chouiyakh introduced Huang Hilbert transform, empirical mode decomposition and instantaneous frequencies into an accurate multicrack identification for a large number of cracks[7]. Zhang used fiber Bragg grating sensors for monitoring the crack of two CFRP concrete composite arches in MTS loading and unloading test. After the test, a method based on Hilbert-Huang Transform was used for determining the cracking time, which take the advantage of Empirical Mode Decomposition[8]. At present, the multi-source excitation research mainly focus on structural response under the multiple incentive parameter combination. But the further research of structural damage is less. Therefore, there is a large research space. 
Traditional electrical sensors in the process of damage detection are difficult to adapt to the operation condition of the aircraft structure environment. Main limitation is that the electricity class sensor resistance to electromagnetic interference ability is poor, poor stability, not easy to decorate, more difficult to achieve the scene of the actual distribution, dynamic, on-line detection . FBG (Fiber Bragg Grating, FBG) has high sensitivity, corrosion resistance, resistance to electromagnetic interference, and it is easy to form multiple parameter distribution sensing array structure etc. Now, many studies have shown that fiber Bragg grating is easy to implement distributed measurement, it can offer a new research direction for the aircraft structure damage detection.

Therefore, this paper proposes a method under double source excitation based on Fiber Bragg grating sensor, taking the board plate as object. And the key contribution is that a double source excitation technique-FBG sensing system is given. The detection method by fiber grating sensing with double source excitation excitation mode is proposed and the influence of frequency, phase parameters have been proposed by envelope analysis is carried out. This paper is structured as follows. Section 2 reviews measuring principle of double source excitation technique and demodulation principle of the FBG is given. Section 3 is devoted to simulation analysis in the double source excitation field of the plate at different time or on different points, whose results lay the foundation for the parameter response characteristic research in section 4 . On this basis, the experimental system is built and the responses of the dual excitations are carried out, and the influence of different parameters are analyzed such as frequency, phase. In Section 5 the experimental data are further analyzed based on envelope analysis for damage detection, which provides basis analysis for damage detection. Section 6 summarizes the full text.

\section{Principle of excitation and reception}

\subsection{Vibration equation of multi source excitation}

The dynamic equation of multi source excitation under low frequency vibration can be expressed as follows:

$$
M \ddot{y}(t)+R \dot{y}(t)+K y(t)=F
$$

where $\mathrm{K}$ is expressed the stiffness matrix, $\mathrm{R}$ is expressed the damping matrix, and $\mathrm{M}$ is expressed the mass matrix. In the formula, $y$ is expressed the displacement vector matrix, $\dot{y}, \ddot{y}$ are expressed the velocity vector matrix and the acceleration vector matrix, and $\mathrm{F}$ represents the external input signal, which is usually used as the acting force. The output signal can be regarded as a linear superposition of the natural mode of vibration.

$$
y(t)=q_{1}\left\{\phi_{1}\right\}+q_{2}\left\{\phi_{2}\right\}+\ldots+q_{n}\left\{\phi_{n}\right\}=\sum_{r=1}^{n} q_{r}\left\{\phi_{r}\right\}
$$


where $q_{r}$ represents the modal coordinate vector, $\phi_{r}$ represents the $r$ order modal shape. Bringing equation (2) to equation (1), the following equation is obtained.

$$
M\left(\sum_{r=1}^{n} \ddot{q}_{r}\left\{\phi_{r}\right\}\right)+R\left(\sum_{r=1}^{n} \dot{q}_{r}\left\{\phi_{r}\right\}\right)+K\left(\sum_{r=1}^{n} q_{r}\left\{\phi_{r}\right\}\right)=F(t)
$$

Assumpting $F(t)=F e^{j w t}, q_{s}=Q_{s} e^{j w t}$, equation (3) can been transform as (4):

$$
\left(-w^{2} m_{s}+j w r_{s}+k_{s}\right) Q_{s} e^{j w t}=\left\{\phi_{s}\right\}^{T} F e^{j w t}
$$

Then the response can be obtained further:

$$
Q_{s}=\frac{\left\{\phi_{s}\right\}^{T} F}{-w^{2} m_{s}+j w r_{s}+k_{s}}
$$

$Q_{s}$ represented as the system response under the force $\left\{\varphi_{s}\right\}^{T} F$, and it can get the following equation according to the formula:

$$
Y=\sum_{r=1}^{N} Q_{s}\left\{\phi_{r}\right\}=\sum_{r=1}^{N} \frac{\left\{\phi_{s}\right\}^{T}\left\{\phi_{r}\right\} F}{-w^{2} m_{s}+j w r_{s}+k_{s}}
$$

The above equation describes the modal model between the vibration of the system response under multi-source excitation and the input force. In general, when the model is used to analyze the multi-source excitation system, the linear time invariant features are used to approximate the model, but the actual model has some nonlinear characteristics, so the solution will affect the accuracy of the model. While the frequency response function, by contrast, is more suitable for the actual analysis of the multi-source excitation test system, which need not too much in advance to predict parameters, and its computational complexity is lower than the time domain model analysis.

When there is noise in the output, there is the best approximation $\hat{H}(w)$ of the frequency response function:

$$
\begin{aligned}
& \hat{H}(w)=\sum_{r=1}^{N} Y_{r}(w) F^{*}{ }_{r}(w)=\sum_{r=1}^{N}\left(H(w) F_{r}(w)+N(w)\right) F^{*}{ }_{r}(w) \\
& =\sum_{r=1}^{N} H(w) F_{r}(w) F^{*}{ }_{r}(w)+\sum_{r=1}^{N} N(w) F^{*}{ }_{r}(w)
\end{aligned}
$$

This scheme focuses on research of dual source excitation, the excitation point is $\mathrm{n}=2$, the equation can be simplified to: 


$$
\begin{aligned}
& \hat{H}(w)=Y_{1}(w) F^{*}{ }_{1}(w)+Y_{2}(w) F^{*}{ }_{2}(w) \\
& =H(w) F_{1}(w) F^{*}{ }_{1}(w)+N(w) F^{*}{ }_{1}(w)+H(w) F_{2}(w) F^{*}{ }_{2}(w)+N(w) F^{*}{ }_{2}(w) \\
& =\sum_{r=1}^{2} H(w) F_{r}(w) F^{*}{ }_{r}(w)+\sum_{r=1}^{2} N(w) F^{*}{ }_{r}(w)
\end{aligned}
$$

By the above formula, the relationship between the input signal and noise is independent. The cross spectrum between the two times the average will tend to zero, then the formula similar to real frequency response function.

\section{2 $\quad$ FBG demodulation principle}

Sensing principle of FBG is based on the Bragg wavelength reflection or transmission spectrum detection. In general, the speed range of fiber Bragg grating demodulation devices is $1 \mathrm{kHz}-4 \mathrm{kHz}$, which do not enough to satisfy the high demodulation speed for ultrasonic signals. So the fiber grating demodulation system need adopt new demodulation methods. The following demodulation system is one of these methods which can satisfy this condition, as shown in figure 2 . The wavelength demodulation system for the FBG sensor is composed of a wavelength tunable laser, an optical coupler and a photodetector. The tunable laser as a light source inspires a beam of light to the FBG through an optical coupler. Then the reflected light from the FBG propagates to the photodetector through the coupler. If the centeral wavelength of the laser beam is tuned to be a little shorter than that of the FBG at free strain, the power relationship between laser and FBG is shown in figure $1[10]$.

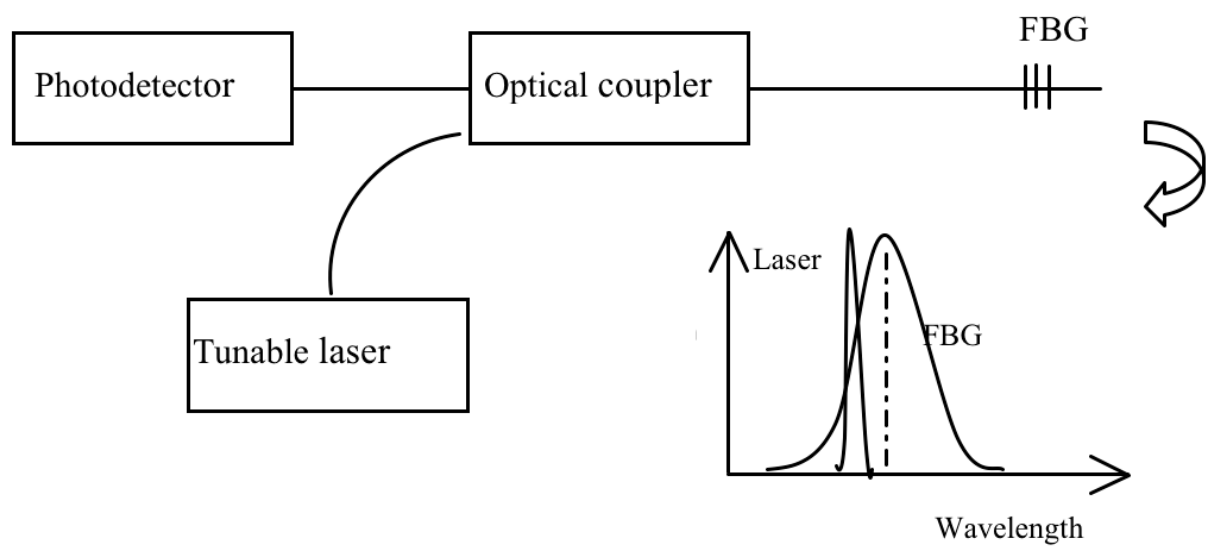

Fig. 1. The demodulation principle of FBG sensor

The optical intensity received by the photodetector is given as following:

$$
P=M_{1} \int_{-\infty}^{\infty} R_{s}(\lambda) S(\lambda) d \lambda
$$


where $R_{s}(\lambda)$ is the reflectivity function of the FBG, $S(\lambda)$ is the spectrum function of the laser source, $M_{1}$ is the efficiency for light energy utilization, which can be regarded as constant if no energy loss. The reflection spectrum of the FBG sensor can be modeled as a Gaussian type to simplify the analysis according to the related model .And the laser beam can also be modeled as Gaussian Beam. So the optical power of photodetector can be derived as following:

$$
P=\frac{M_{1}}{2} R_{s 0} I_{0} \sqrt{\frac{\pi}{\ln 2}}\left\{\frac{\Delta \lambda_{0} \Delta \lambda_{S}}{\left(\Delta \lambda_{0}^{2}+\Delta \lambda_{S}{ }^{2}\right)^{\frac{1}{2}}} \times \exp \left[-4 \ln 2 \frac{\left(\lambda-\lambda_{S}\right)^{2}}{\Delta \lambda_{S}{ }^{2}+\Delta \lambda_{0}^{2}}\right]\right\}
$$

where $R_{s 0}$ is the maximum reflectivity, $\lambda_{s}$ is the center wavelength of the FBG, $\Delta \lambda_{s}, \Delta \lambda_{0}$ is its spectral full width at half maximum. $I_{0}$ is the peak power, $\lambda_{0}$ is the center wavelength of the laser beam. Therefore, the optical power of photodetector can be obtained according to Eq.(10). The optical power has the maximum if the center wavelength of the FBG equals to that of the laser. When FBG is compressed, there will be little change in wavelength of the FBG in this case. And the overlapped region between the optical power of the laser beam and that of FBG will change along with the intensity of output light accordingly. Thus the wavelength change of the FBG can be measured by the intensity of the photodetector. Therefore, it can analyze the axial strain change of fiber Bragg grating by detecting its wavelength drift, avoiding the influence of electromagnetic field.

\section{Simulation analysis of double vibration}

\subsection{Frequency domain simulation}

Finite element analysis can be used in computer software, and its powerful analysis function is used for numerical analysis. Here, ANSYS finite element software is used to analyze the structure of the plate, and the corresponding natural frequencies are obtained. The thin plate model is established, and the plate size is $500 \times 500 \times 1 \mathrm{~mm} 3$, as shown in Figure 2 (a). Then the properties of the aluminum plate are set after selecting the elastic shell element shell63: density is $2700 \mathrm{~kg} / \mathrm{m}^{3}$, elastic modulus is $72 \mathrm{GPa}$, Poisson's ratio is 0.3 . The constraint is applied to the four corners of the thin plate, and the full displacement constraint condition is selected. After solving the mesh, the natural frequencies of the first 10 orders are obtained as shown in table 1:

Table 1. the natural frequency of the first ten orders of aluminum plate

\begin{tabular}{ccccccccccc}
\hline orders & 1 & 2 & 3 & 4 & 5 & 6 & 7 & 8 & 9 & 10 \\
\hline $\mathrm{f}(\mathrm{Hz})$ & 14.7 & 29.3 & 29.9 & 36.2 & 68.3 & 71.2 & 83.9 & 85.2 & 114.1 & 129.0 \\
\hline
\end{tabular}


On the basis of the above model, the harmonic response characteristics of the plate under the same double vibration excitation of the two parameters are studied based on the formula (8). Harmonic response analysis of the excitation using two same frequency sinusoidal signal, applied on $\mathrm{B}, \mathrm{A}$ in the center of the thin plate, whose coordinates is: $(210,250),(290,250)$. Detection points are set to P1-P4 four points, the coordinates are $(190,250),(170,250),(150,250),(130,250)$, as shown in Figure 2.

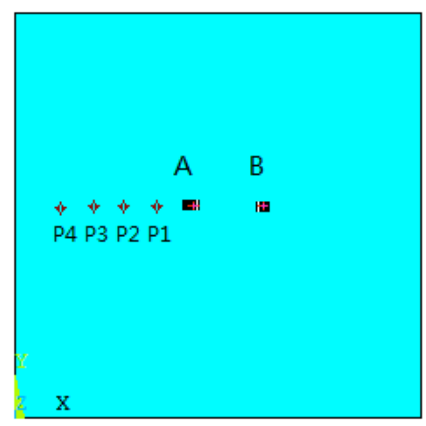

(a) Geometric model

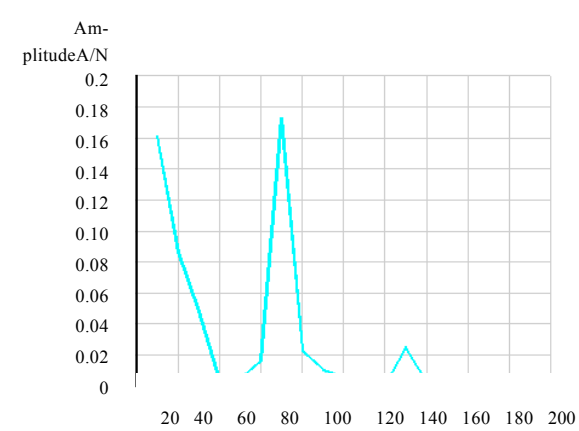

(b) Detecting position P1

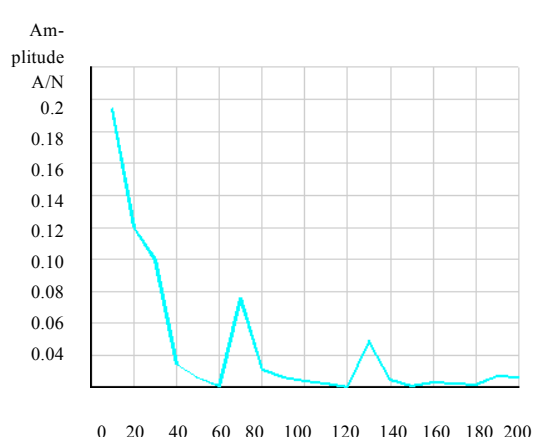

(d) Detecting position P3

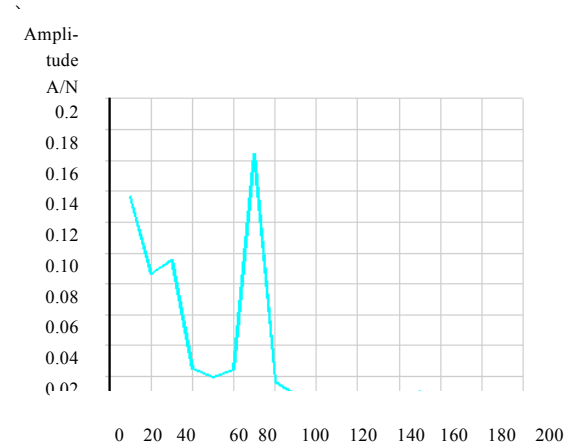

(c) Detecting position P2

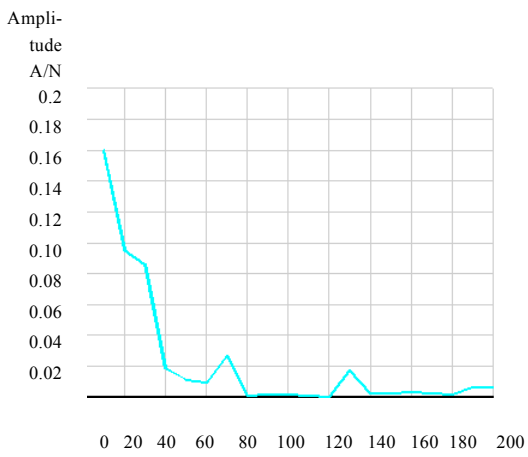

(e) Detecting position P4

Fig. 2. Testing point of harmonic response 
Harmonic load is applied by setting the input amplitude, initial phase, the frequency of the applied load range. ANSYS solver is selected harmonic response analysis by default the frontal solver. Among them, the amplitude of harmonic load is set through the real part and imaginary part form respectively, here it is set $10 \mathrm{~N}$ sinusoidal amplitude, which corresponds to the real part and the imaginary part are $7.07 \mathrm{~N}$ and initial phase angle is $\pi / 4$, the frequency range is set $0-200 \mathrm{~Hz}$ by choice of step growth gradually. Finally, harmonic response curves are obtained on the testing points under two sine excitations, as shown in Figure 2 (b) - (e).

From 2 (b) - (e) we can know that there are more obvious response amplitudes of the plate structure near the frequency of $30 \mathrm{~Hz}, 70 \mathrm{~Hz}, 130 \mathrm{~Hz}$, which correspond to the third order, sixth order and tenth order natural frequency of the plate structure. Among them, P2 and P1 are near two excitation sources, and the response amplitude is larger, while P3 and P4 are far away from the excitation sources, and the response amplitude is relatively smaller. Therefore, in double vibration process, excitation frequency parameters can be selected at the natural frequency of the plate, which makes the response amplitude becomes larger; in addition, due to the limited range of incentive, the detection position should not too far from the excitation point. According to the harmonic load under the action of two-dimensional strain response characteristics, this is provide theoretical basis for subsequent double vibration sensing distribution.

\subsection{Time domain simulation}

The vibration signal is less than $20 \mathrm{kHz}$, assuming two low-frequency vibration signals $\mathrm{x} 1, \mathrm{x} 2$ respectively, sine wave, amplitudes respectively $50 \mathrm{~V}$ and $20 \mathrm{~V}$. According to the double vibration incentive theory, the response of the detection signal has the following characteristics: the signal frequency will be consistent to the excitation frequency, the detected amplitude just changes. Here assuming that the amplitude attenuation coefficient is 10 , according to the transmission characteristics of the system, the signal amplitudes of the fiber Bragg grating detection are corresponding $2 \mathrm{~V}$ and $5 \mathrm{~V}$ respectively by the common effect under two source of vibration excitation as shown in Figure 3: In figure 3 (a), excitation frequencies are respectively $10 \mathrm{kHz}, 2$ $\mathrm{kHz}$. For low frequency and large amplitude vibration signals, there is a sawtooth sine signal in superimposed signal after superposition of high frequency signals of small amplitude, but the overall trend shows the low frequency vibration characteristics of the large amplitude.

While figure 3 (b), excitation frequencies are respectively $10 \mathrm{kHz}, 9 \mathrm{KHz}$. After two superposition of vibration signal, because the frequency is relatively close, there is an obvious phenomenon: it makes the stacked waveform.

In Figure 4(a), the excitation signal $\mathrm{x} 1, \mathrm{x} 2$ frequencies are respectively $10 \mathrm{kHz}$, $9 \mathrm{KHz}$, the amplitude parameters are the same as the front figure 3. Accordingly, the response frequency is $10 \mathrm{kHz}$ and $9 \mathrm{kHz}$, too. On the basis of this, change the initial phase of the second vibration $\mathrm{x} 2$ is respectively $1,2,3$ radians, which makes the two wave initial phase difference changes. It can seen that the maximum position changes, the envelope gradually shifts to the right. But the cycle of the shoot unchangs. In 
Figure 4 (b), keeping the frequency of the first vibration signal $\mathrm{x} 110 \mathrm{kHz}$ unchanged, the frequency of the second vibration signal $x 2$ is changed In turn $9.5 \mathrm{kHz}, 7.5 \mathrm{kHz}$, $2.5 \mathrm{kHz}$. It can be seen that the frequency difference of the two vibration source signals is smaller, the period of the beat is larger; on the contrary, the greater the frequency difference is, the smaller the cycle is.
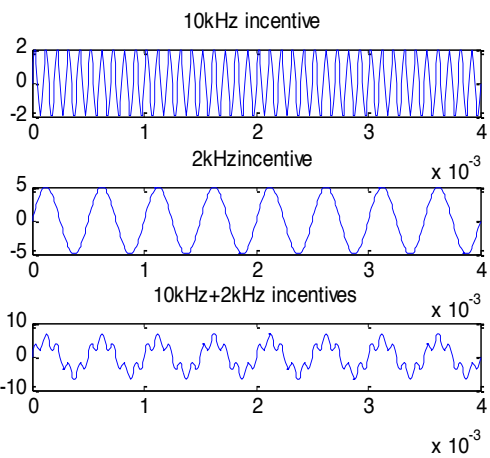

(a) With big frequency difference

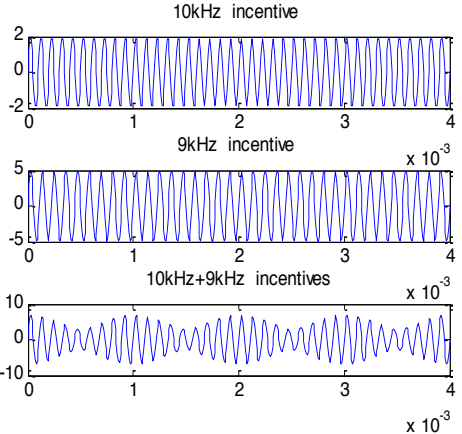

(b) With small frequency difference

Fig. 3. Vibration superposition responses under the different combination
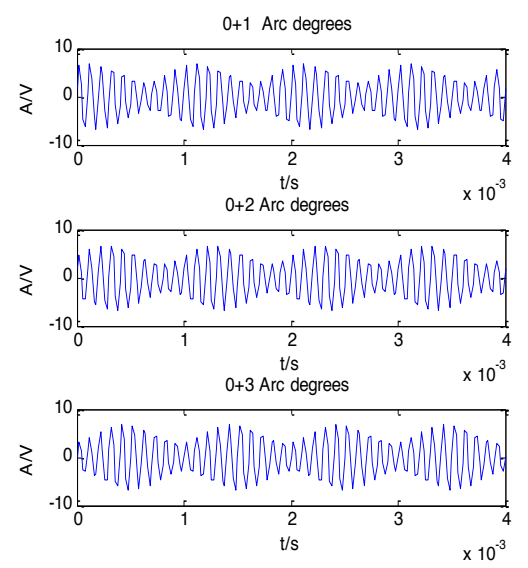

(a) Change the initial phase
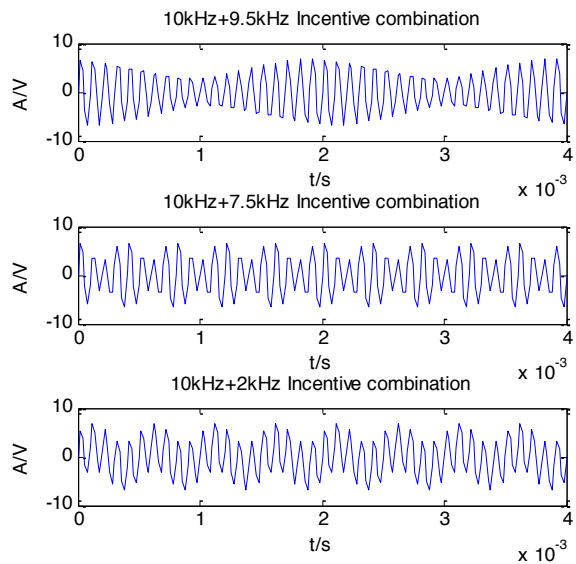

(b) Change the low frequency

Fig. 4. Vibration superposition

According to the above simulation analysis, it can be seen that if two after vibration superposition harmonic vibration frequency is very close, there will be a complicated vibration meter. In order to avoid mutual coupling effect of two vibration sources, it is suitable for the excitation signal selection frequency difference is bigger, at least more than 5 times. At this time, The coupling effect is weak, the overall change can reflect the change of the low frequency component characteristics. This is advantageous for the vibration source excitation signal detection analysis. 


\section{Double vibration experiment system}

In order to verify the double vibration under the excitation of the output response characteristics, we use adjustable laser edge filter demodulation as new demodulation system. Principle diagram is shown in figure 5 (a), where piezoelectric wafers are used for the excitation, while FBG sensors are used for detection response.

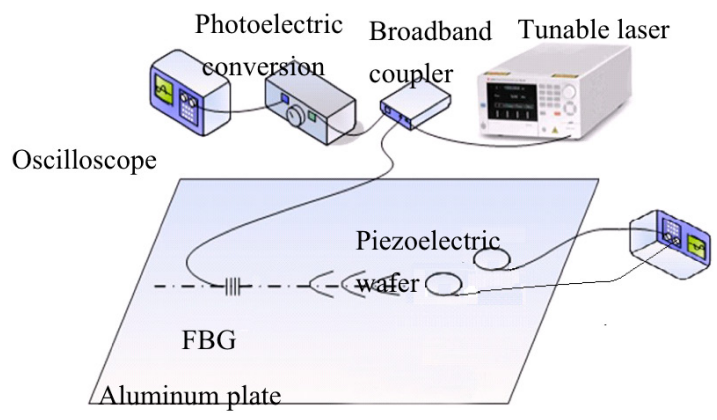

(a) Experimental system

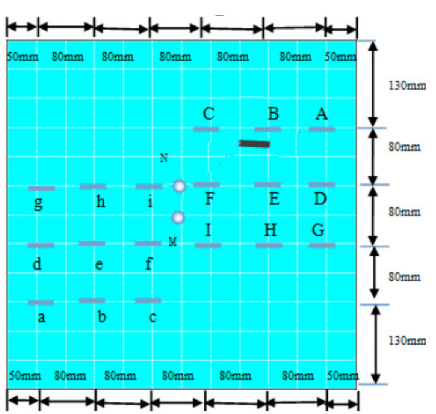

(b) Component layout

Fig. 5. Experimental system and layout

Here taking thin aluminum plate structure $(500 \mathrm{~mm} * 500 \mathrm{~mm} * 1 \mathrm{~mm})$ as object, two source of vibration excitations are applied on piezoelectric wafers. M, N, represent respectively the piezoelectric wafers with the thickness of $0.5 \mathrm{~mm}$, the diameter of $10 \mathrm{~mm}$. The horizontal lines represent the fiber grating Grating sensors. The crack is located in the upper right corner, whose size is $20 \mathrm{~mm} \times 2 \mathrm{~mm} \times 1 \mathrm{~mm}$. FBG arrangement in the healthy area are represented by A - I respectively, while FBG arrangement in the damaged area are represented by $\mathrm{a}-\mathrm{i}$. The specific arrangement is shown in figure 5 (b). Here, parameters affect such as frequency and phase of the excitation signal are first analyzed by comparison with simulation results. Then the peak strains are obtained by envelope analysis, and last the crack position are discussed.

Keeping the frequencies are $8.4 \mathrm{kHz}, 8.6 \mathrm{kHz}$, we change the initial phase of $\theta_{D}: 0$, $60,120,180$ degrees, then get the results as shown in figure 6 , which shows that the excitation frequency is closer, the beat phenomenon is more obvious with the change of the initial phase, and the position of the maximum value is changed; but the cycle of the beat is unchanged. This phenomenon is consistent with the theoretical analysis.

Keeping one excitation frequency $f_{a}$ of $10.5 \mathrm{kHz}$, another frequency $f_{b}$ is in turn changed to $10.4,10.9,9,8.5 \mathrm{kHz}$ and the combined response result is shown in figure 7. From the graph 7 , it can seen that the frequency difference between the two signals is smaller, the cycle is larger. This phenomenon is consistent with the theoretical analysis. 


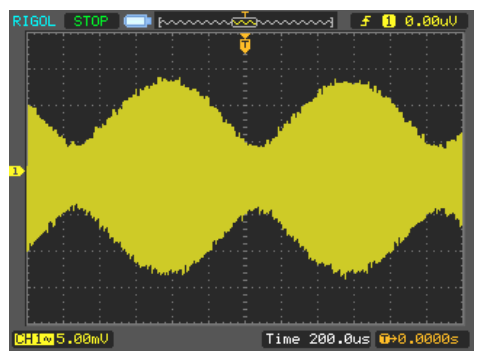

(a) $\theta_{D}=0$

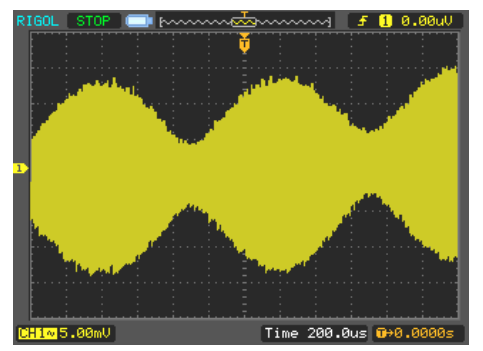

(c) $\theta_{D}=120$

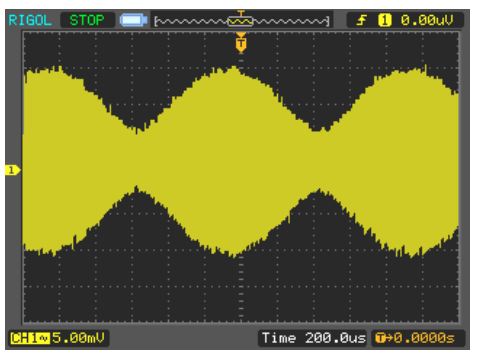

(b) $\theta_{D}=60$

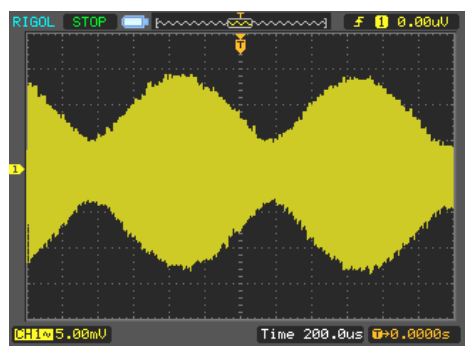

(d) $\theta_{D}=180$

Fig. 6. Vibration responses under different phases

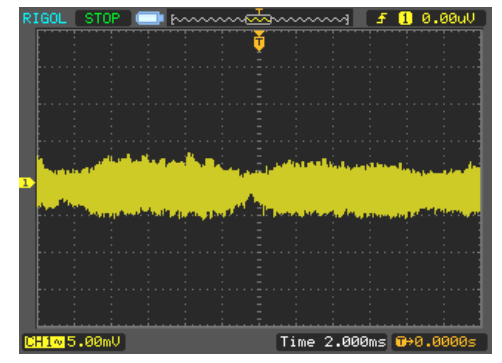

(a) $f_{b}=10.4 \mathrm{kHz}$

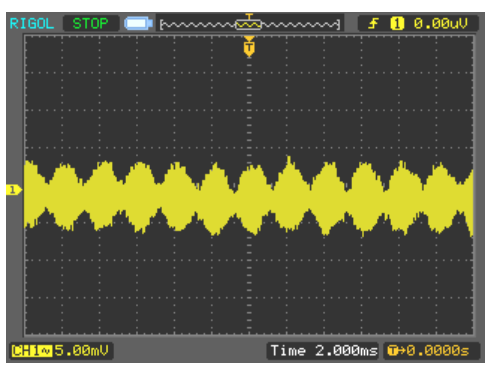

(c) $f_{b}=9 \mathrm{kHz} 0$

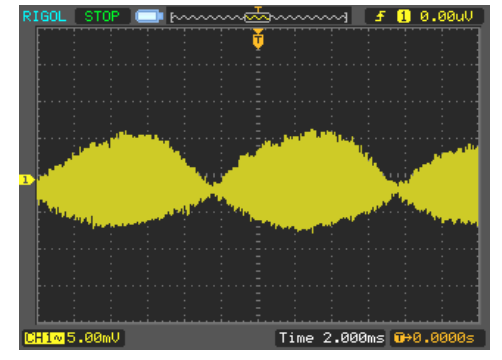

(b) $f_{b}=10.9 \mathrm{kHz}$

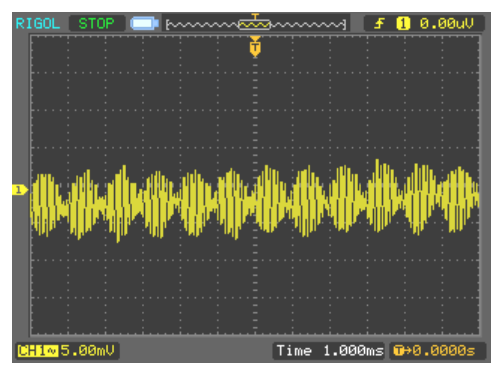

(d) $f_{b}=8.5 \mathrm{kHz}$

Fig. 7. Vibration responses under different frequencies 


\section{$5 \quad$ Envelope analysis}

On the basis of the double vibration experiment data, envelopment analysis is carried out on the double vibration signal in two cases. Keeping one excitation frequency, change another excitation frequency in turn, $10.4 \mathrm{kHz}, 10.9 \mathrm{kHz}, 9 \mathrm{kHz}$. The corresponding amplitude spectrums are first obtained by Fourier transform, as shown in figure 8(a)- (c), then envelope spectrums are obtained by using the Hilbert transform envelope and the detection envelope, as shown in figure 8(d), (e). It can be seen that the envelope is suitable for observation overall trends under different combination incentive.

In figure 8 (a), two frequency $10.4 \mathrm{kHz}, 10.5 \mathrm{kHz}$ difference is minimum, and the beat period is the longest; In figure 8 (b), two frequency $10.9 \mathrm{kHz}, 10.5 \mathrm{kHz}$ frequency difference is big, the beat phenomena is also appeared, the beat cycle is short; in figure 8 (c), two frequency $9 \mathrm{KHz}, 10.5 \mathrm{kHz}$ frequency difference is the biggest, the single beat is the the shortest. It can be seen, two of the excitation signal frequency difference is small, beat cycle is bigger. In addition, due to the signal noise will affect the result to a certain extent of the spectrum of the envelope, such as figure 8 (a) due to the obvious noise signal, the envelope spectrum in addition to the two sources of vibration signals, other components $6.25 \mathrm{khz}, 2 \mathrm{kHz}$ signal clutter wave frequency components. And figure 8 (b) and figure 8 (c) in the noise signal is very small, the envelope spectrum contains two main vibration signal source frequency, other clutter components can be ignored. Contrasting the envelope waveform in figure 8 (d), figure 8 (e), it can be seen that general rule changes of two envelope, but in the local details, it is closer to the contour of the output waveform by Hilbert transform envelope.

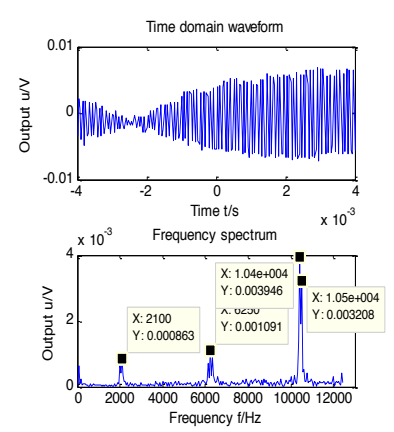

(a) $10.4 \mathrm{kHz}$

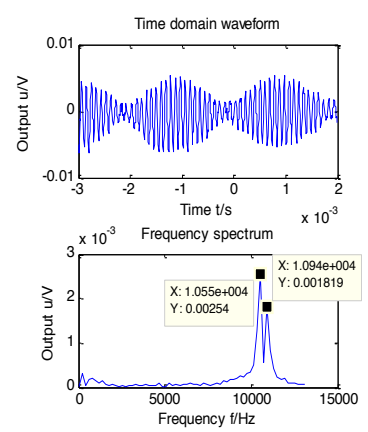

(b) $10.9 \mathrm{kHz}$

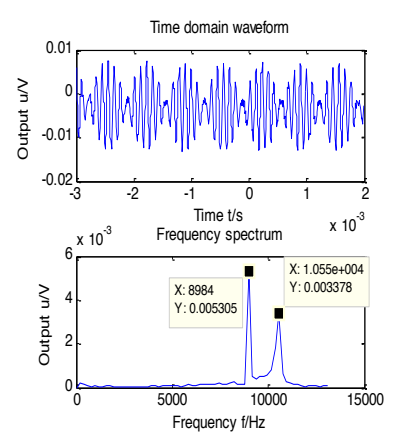

(c) $9 \mathrm{kHz}$ 

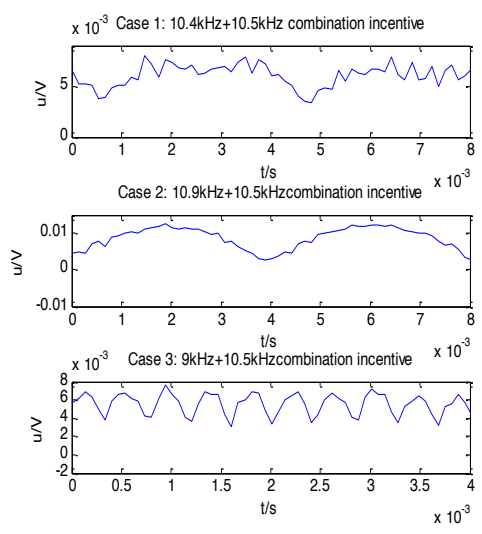

(d) Hilbert envelope
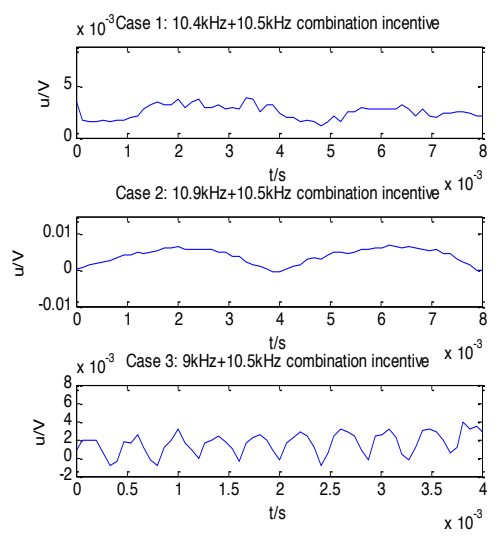

(e) Detection envelope

Fig. 8. Amplitude spectrums under different frequency combinations

Use the same method of phase change, and Figure 9 is keeping two excitation frequency $8.4 \mathrm{kHz}, 8.6 \mathrm{kHz}$ invariable premise, double vibration signal envelope spectrum of different phase combination, early phase combination in turn is 0 and 0,0 degrees and 60 degrees, 0 degrees and 120 degrees. First the corresponding amplitude spectrums are obtained after Fourier transform, as shown in Figure 9 (a) - (c), then envelope spectrums using Hilbert transform envelope and detection envelope are obtained as shown in Figure 9 (d), (e).

By comparing the amplitude spectra of figure 9 (a), (b), (c), the peak frequency in the spectrum is correspond to the excitation frequency of $8.4 \mathrm{kHz}$ and $8.6 \mathrm{kHz}$. Because the two excitation frequency is close, there is a beat phenomenon in the superposition signal: beat cycle is maintained but the position of the maximum value will change with the initial phase difference changes, which can be found from figure 9 (d), (e). By comparing the two envelope characteristics found that the two envelope methods broadly consistent with the overall variation, but in the local details, it is closer to the contour of the output waveform by Hilbert transform envelope, which makes the envelope to extract more complete. Hilbert transform to extract the signal envelope method is suitable for double vibration signal and has certain antiinterference ability.

According to the parameter analysis results, in order to avoid the influence of frequency and phase parameters, here the same frequency and phase of sinusoidal signals are selected as excitation signals, focusing on the response signal analysis of fiber Bragg grating sensors: Incentive frequency are $60 \mathrm{~Hz}$, phase are 0 . The peak changes are obtained shown in table 2 by comparing the peak strains of FBG response signals in the health area and damage area. Then the corresponding histograms are shown in figure 10, from which it can be seen that the highest peak position is roughly corresponding to the crack position. 
Paper-Response Analysis of a Plate Structure under Double Source Excitation by FBG Sensors
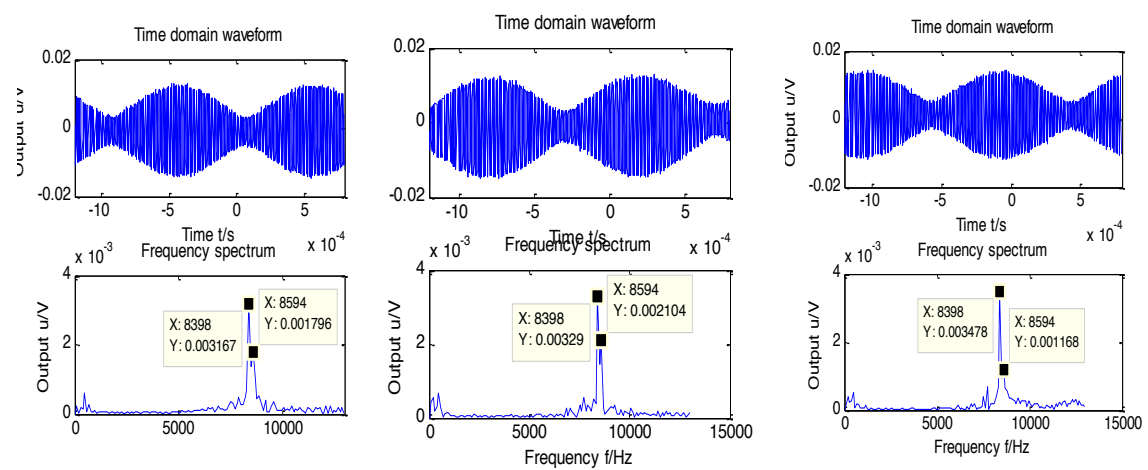

(a) $0+0$

(b) $0+60$

(c) $0+120$
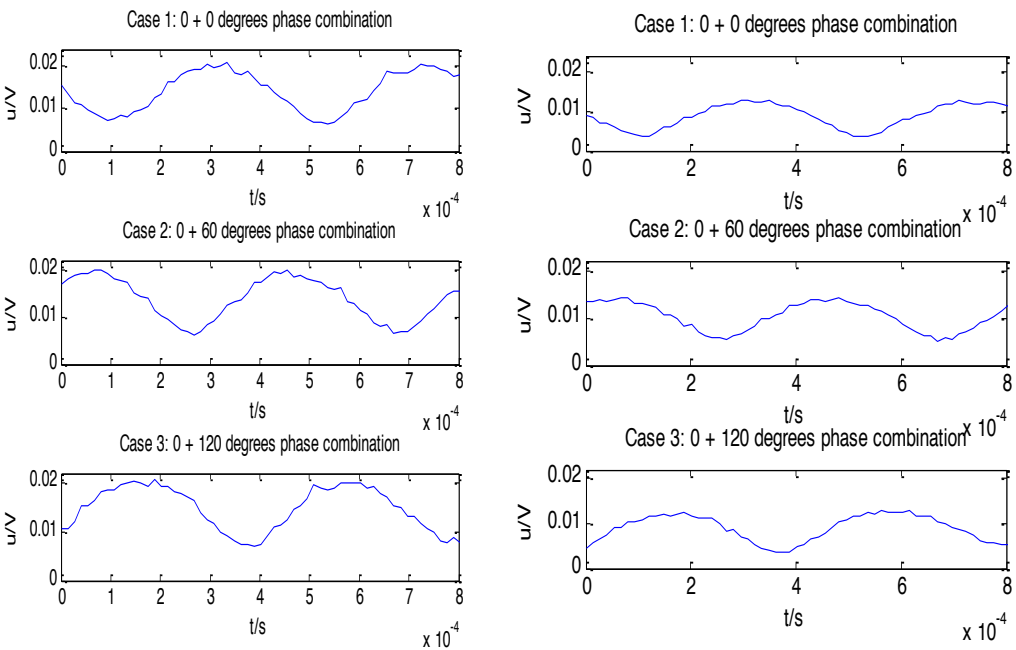

(d) Hilbert envelope

(e) Detection envelope

Fig. 9. Amplitude spectrums under different phase combinations

Table 2. Peak detection under double excitations

\begin{tabular}{cccccccccc}
\hline \multirow{2}{*}{$\begin{array}{c}\text { FBGs/ Peak } \\
\text { strain }(\boldsymbol{\mu} \boldsymbol{\varepsilon})\end{array}$} & $\mathbf{A} / \mathbf{a}$ & $\mathbf{B} / \mathbf{b}$ & $\mathbf{C} / \mathbf{c}$ & $\mathbf{D} / \mathbf{d}$ & $\mathbf{E} / \mathbf{e}$ & $\mathbf{F} / \mathbf{f}$ & $\mathbf{G} / \mathbf{g}$ & $\mathbf{H} / \mathbf{h}$ & $\mathbf{I} / \mathbf{i}$ \\
\cline { 2 - 12 }$y$ & 1285 & 1287 & 1292 & 1290 & 1300 & 1301 & 1306 & 1315 \\
\hline Healthy area & 10 & 10 & 24 & 4 & 8 & 16 & 5 & 5 & 5 \\
\hline Damaged area & 9 & 14 & 23 & 4 & 13 & 4 & 5 & 4 & 15
\end{tabular}




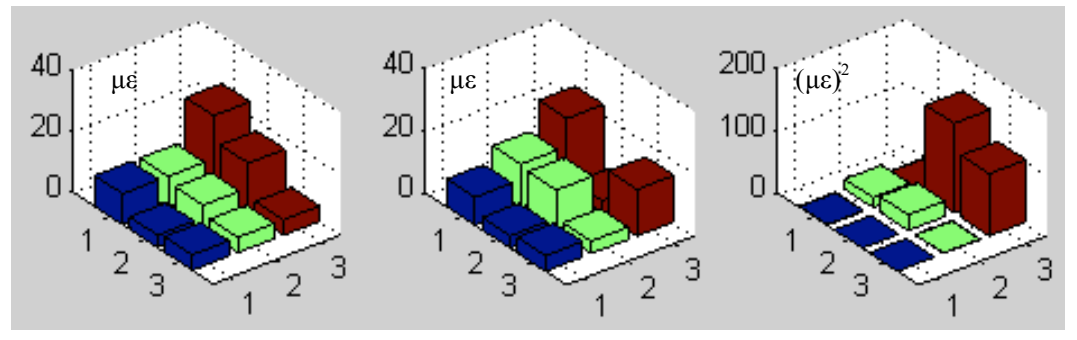

(a) Damaged area

(b) Healthy area

(c) Difference

Fig. 10.The peak response histograms under the same motivation parameters

\section{Conclusion}

This work focuses on response analysis of plate structure under double source excitation by FBG, taking the plate structure as the object, a new attempt by double source excitation detection with FBG sensors has been investigated. First, double source excitation technique and demodulation system of FBG sensors are introduced. Second, the simulation analysis under the double source excitation are carried out and frequency domain simulation of the plate structure are performed, which reveal various signals under double source excitation on different detection positions. Third, the experimental system based on double source excitation -fiber Bragg grating detection is built and response analysis of FBG sensors under the different excitations are given. On this basis, this double source excitation-FBG sensing technique based on envelope analysis is applied. The results reveal an efficient double source excitation technique by FBG sensors, whose results show that it is feasible to crack damage detection.

\section{$7 \quad$ Acknowledgment}

This work is supported the following fund:

2017 The project of Hubei Province Natural Science Foundation for Youths (No.2017CFC890).

2017 the Science Research Project of Hubei Province Education Department (No.B2017298).

\section{$8 \quad$ Reference}

[1] Wieslaw Ostachowicz, Pawel Kudela, Pawel Malinowski, Tomasz Wandowski: Damage localisation in plate-like structures based on PZT sensors. Mechanical Systems and Signal Processing, 23, 1805-1829, (2009). https://doi.org/10.1016/j.ymssp.2008.10.011

[2] Wang D, Ye L, Lu Y, et al: A damage diagnostic imaging algorithm based on the quantitative comparison of Lamb wave signals[J]. Smart Materials and structures, 19(6), 1-12, (2010). https://doi.org/10.1088/0964-1726/19/6/065008 
[3] Jeng-Wen Lin: Hilbert-Huang Transform Based Modal Analysis of Structures. ASME 2008 Pressure Vessels and Piping Conference, Seismic Engineering, Vol. 8, 123-131, (2008).

[4] R Kashyap, TR Lenka,S Baishya: A Model for Doubly Clamped Piezoelectric Energy Harvesters With Segmented Electrodes. IEEE Electron Device Letters. 36 (12), 13691372, (2015). https://doi.org/10.1109/LED.2015.2496186

[5] S Adhikari, MI Friswell, DJ Inman: Piezoelectric energy harvesting from broadband random vibrations. Smart Materials \& Structures. 18 (11) , 269-273, (2009). https://doi.org/10.1088/0964-1726/18/11/115005

[6] TianLi Huang, MengLin Lou, HuaPeng Chen, NingBo Wang: An orthogonal HilbertHuang transform and its application in the spectral representation of earthquake accelerograms. Soil Dynamics and Earthquake Engineering. 378-389, (2018). https://doi.org/10.1016/j.soildyn.2017.11.005

[7] H. Chouiyakh, L. Azrar, K. Alnefaie, O. Akourri: Vibration and multi-crack identification of Timoshenko beams under moving mass using the differential quadrature method. 1-11, (2017).

[8] Qinghua Zhang, Yuan Wang, Yangyang Sun, Lei Gao, Yin Yue. Hilbert-Huang Transform based method for monitoring the crack of concrete arch by using FBG sensors. Optik International Journal for Light and Electron Optics , 127 (6) , 3417-3422, (2016 ). https://doi.org/10.1016/j.ijleo.2015.12.087

\section{Authors}

CAI LI ${ }^{(*)}$ (1980-), come from Wuhan, Hubei province. Ph.D.degree, the main research direction for equipment detection and diagnosis.(e-mail: caii_whut@163.com, affiliation: School of Mechanical and Electronic Engineering, Wuhan Donghu University, Wuhan, 430070, China).

HUANG XIAO(1982-), come from Wuhan, Hubei province. Ph.D.degree, the main research direction for fiber Bragg grating detection technology. (e-mail: 51277619@qq.com, affiliation: School of Mechanical and Electronic Engineering, Wuhan University of Technology, Wuhan, 430070, China).

MENG LIJUN (1983-), come from Wuhan, Hubei province. Ph.D.degree, the main research direction for ultrasonic detection and fiber sensing technology. (e-mail: 35215584@ qq.com, affiliation: School of Electromechanical and Civil Engineering, Jianghan University, Wuhan, 430070, China).

Article submitted 23 January 2018. Resubmited 15 March 2018. Final acceptance 07 May 2018. Final version published as submitted by the authors. 